\title{
Laser interference lithography with highly accurate interferometric alignment
}

\author{
Frank J. van Soest, Henk A.G.M. van Wolferen, Hugo J.W.M. Hoekstra, René M. de \\ Ridder, Kerstin Wörhoff and Paul V. Lambeck \\ University of Twente, MESA ${ }^{+}$Research Institute, \\ Integrated Optical MicroSystems group (IOMS), Postbus 217, 7500 AE Enschede, the Netherlands
}

\begin{abstract}
Three dimensional photonic crystals, e.g. for obtaining the so-called woodpile structure, can, among others, be fabricated by vertical stacking of multiple gratings. One of the requirements for obtaining a full photonic bandgap in such a photonic crystal is an accurate angular and lateral alignment of the successive gratings. Using laser interference lithography at $266 \mathrm{~nm}$ wavelength, we fabricated gratings in silicon with periods down to $300 \mathrm{~nm}$. We present a method for aligning further grating exposures with respect to this grating with a 0.001 degree angular and a few nanometers lateral resolution.
\end{abstract}

\section{Introduction}

The down-scaling of integrated optical (IO) devices is of importance for both theoretical and practical reasons and has attracted much research. A major step forward in this field was the discovery of photonic crystals [1], which are periodic structures in space (refractive index lattices) with a period in the order of the wavelength of light. These structures, which may prohibit the propagation of light in any direction for a certain wavelength range, the so-called photonic bandgap, provide new opportunities for developing ultra compact key components for integrated optics, such as splitters, wavelength filters, delay lines and light sources, thus promising a size reduction of IO circuits by orders of magnitude. Several requirements regarding lattice symmetry, refractive index contrast and microstructure should be satisfied in order to obtain a full 3D photonic bandgap.

One promising way to realize a 3D photonic crystal, is by piling submicron spheres, which self-organize into an (inverse) opal structure [2]. Another promising technique concerns the oblique deposition on a rotating substrate. This may yield spiral structures with the potential to have a relatively large bandgap [3]. The controlled introduction of arbitrary lattice defects (which act as functional structures like waveguides, etc.) in this type of structures is far from obvious. However, lithographic fabrication of photonic crystals in a layer by layer fashion provides a natural way for defining these defects.

A Photonic Crystal ( $\mathrm{PhC})$ structure that is especially

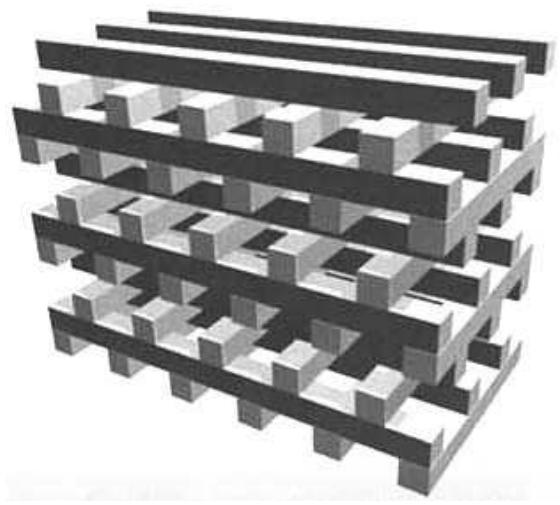

Figure 1: Example of a woodpile structure [1]. well suited for lithographic fabrication is the 3-Dimensional Woodpile Structured Photonic Crystal (WPC) (see Figure 1). This structure has been proposed for the first time in 1994 [4]. Since that time, four-layer structures with a stop band between 1.35 and $1.95 \mu \mathrm{m}$ [5], eight-layer structures, created with the use of wafer bonding [6], and even waveguides for a wavelength of $\pm 8 \mu \mathrm{m}$ in a woodpile structure, have been demonstrated. 
For the lithographic production of 3D PhC's, a sufficiently accurate process is required. Conventional mask lithography does not provide the required resolution and alignment accuracy because the typical structure size is less than $500 \mathrm{~nm}$ for PhC's with a bandgap around $1550 \mathrm{~nm}$, in a high-index material like silicon. Using expensive equipment for deep-UV or (relatively slow) direct e-beam writing lithography, this problem can be overcome. As an alternative, Laser Interference Lithography (LIL) offers an excellent, relatively easy and cost-effective technique for the production of periodic structures.

However, alignment of these structures is essential, as has been shown for WPC's [7]. For these structures, the thickness and the width of the bars are very relevant as well, but they have significantly less impact than misalignment [7]. The influence of a deviation of $20 \%$ from the optimum thickness and width of the bars, results in a bandgap reduction of $18 \%$ and $15 \%$ respectively. On the other hand, misalignment in one direction by $20 \%$ of the lattice period, gives already a bandgap reduction of $44 \%$, and $20 \%$ misalignment in two directions reduces the bandgap even by $58 \%$.

\section{Laser Interference Lithography}

LIL is a maskless lithographic technique using the interference pattern of two obliquely incident beams. We used a setup based on the Lloyds interferometer, schematically depicted in Figure 2 , where a regular pattern is produced by interference of a laser beam and a "second" beam. Because the second beam is created by a mirror very close to the substrate, the setup is less sensitive to vibrations than a true dual beam interference setup. The period of the interference

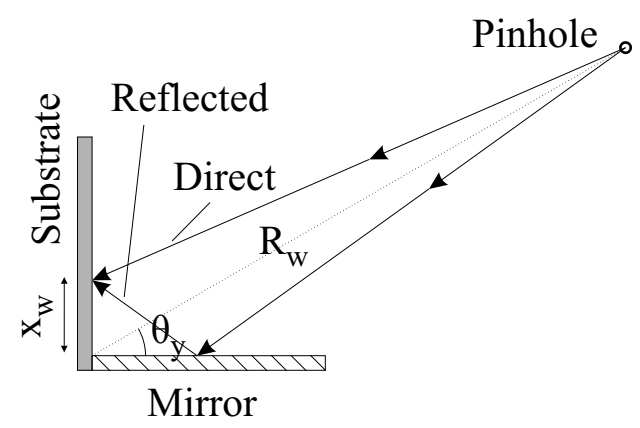

Figure 2: Lloyds interferometer [8]. pattern, and thus that of the grating recorded in the resist layer on the substrate, is given by: $\Lambda_{I P}=\lambda /(2 \sin \theta)$. With a $266 \mathrm{~nm}$ light source, periods of $150 \mathrm{~nm}$ to $500 \mathrm{~nm}$ can easily be made. For smaller periods, however, the quality of the development process and thickness of the resist have to meet some severe requirements because of the viscous character of the development liquid at submicron scale. After illumination and development of the resist, the grating can be transferred to the substrate by, e.g. reactive ion etching.

\section{Alignment}

As mentioned before, the geometry of the structure is of major importance in order to produce a photonic crystal having a full bandgap. When a grating is made with the LILsetup, it consists of several tens of thousands of lines. This relatively large size of the grating imposes very strict requirements on alignment.

For the stacking of, e.g. WPC's, this means that the lines of every second next layer have to be parallel within a very high degree and the periods of the gratings should be very well matched, in order to satisfy the conditions for a full 3D bandgap. To realize this, interferometric positioning can be used, as explained below.

The presented interferometric positioning method is based on analysis of the interference of two almost identical wavefronts [9] at two spots, next to the input pinhole (see Figure 
4). The first (second) spot originates from the interference of the first order diffraction of the direct (reflected) beam and the zeroth order diffraction of the reflected (direct) beam. When the substrate with grating is at exactly the same position as during the production of the grating, the two wavefronts at either one of the spots, coincide exactly, yielding two relatively homogenous spots on a fluorescent screen at both sides of the pinhole. Even a very small misalignment of the substrate gives rise to a deviation in the orientations of the two wave fronts, producing, in a plane wave approximation, fringe patterns with periods in the horizontal and vertical directions $W_{x}$ and $W_{y}$ respectively, given by [8]:

$$
W_{x}=\frac{\lambda}{2 \Delta \theta_{y} \cos \theta_{y}} \quad W_{y}=\frac{\lambda}{2 \Delta \theta_{z} \sin \theta_{y}}
$$

Here, $\lambda$ is the wavelength of the used light, $\Delta \theta_{y}$ and $\Delta \theta_{z}$ are the misalignment angles and $\theta_{y}$ the angle at which the grating was made (shown in Figure 2).

The strong dependence of the fringe pattern on the misalignment is shown in Figures 3. Here, a grating with a period of $266 \mathrm{~nm}$ was re-aligned. The accuracy of realignment was limited by the resolution of the rotation stages, which is $0.001^{\circ}$. The radius of curvature (here being $2.3 \mathrm{~m}$.) and the sample quality are also important here, as can be seen from the distortions of the patterns in Figures 3.

In addition to the rotational realignment, also accurate positioning in lateral direction is of utmost importance. For WPC's, every second next layer is shifted laterally by half a period with respect to the preceding layer. After angular alignment, the intensity of the fringe spots still depends on the phase

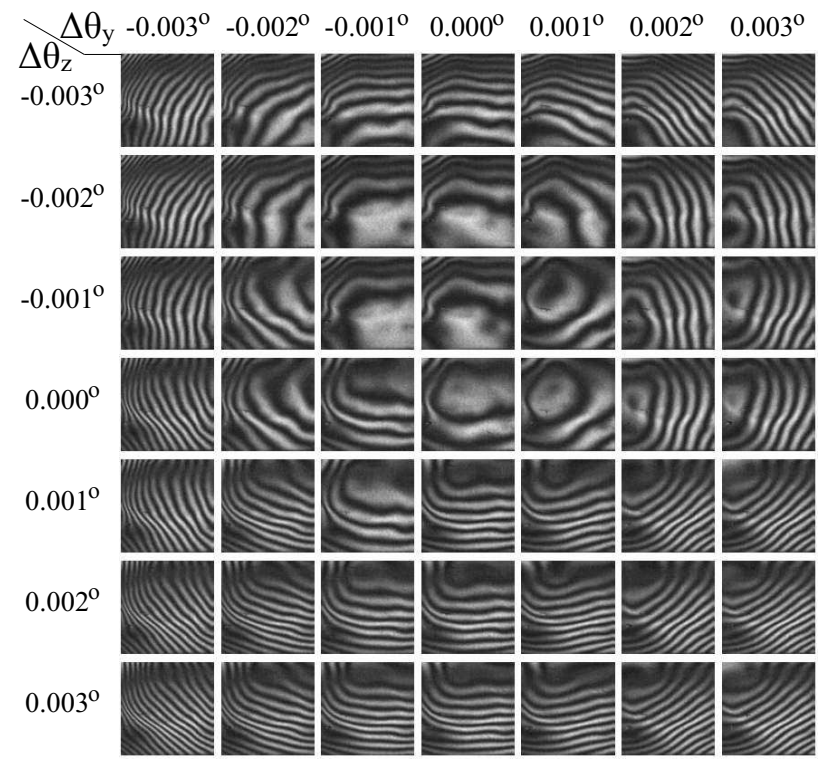

Figure 3: 7x7 matrix of observations of the fringe pattern for different deviation angles with respect to the original alignment. [8].

shift between the two wave fronts caused by the lateral shift of the grating. Figure 5 shows a measurement (after angular alignment) of the intensity of the center of the fringe spot versus the lateral shift. This shift was realized by a piezo driven translation stage with a resolution of $1 \mathrm{~nm}$. The irregularities in the figure are caused by power fluctuations of the laser beam. Relative measurement of the spot intensity with respect to the beam power will give better results. The high sensitivity of the spot power as function of the grating translation is, however, very clear.

\section{Conclusions}

It can be concluded that interferometric positioning offers good possibilities for aligning a structure sufficiently accurate to make 3D PhC's. The realized angular re-alignment accuracy of $0.001^{\circ}$ was limited by the resolution of the rotation stages. With better rotation stages and better samples, an improvement in re-alignment accuracy with one order of 


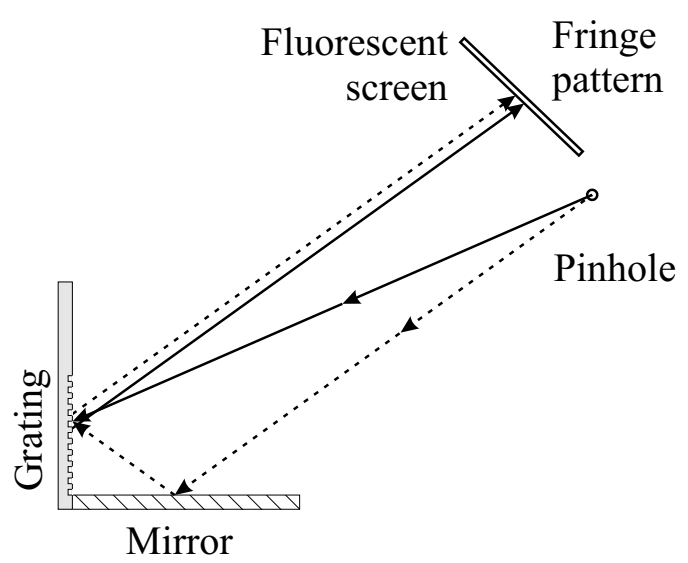

Figure 4: Principle of alignment; matching reflected en diffracted wavefronts.

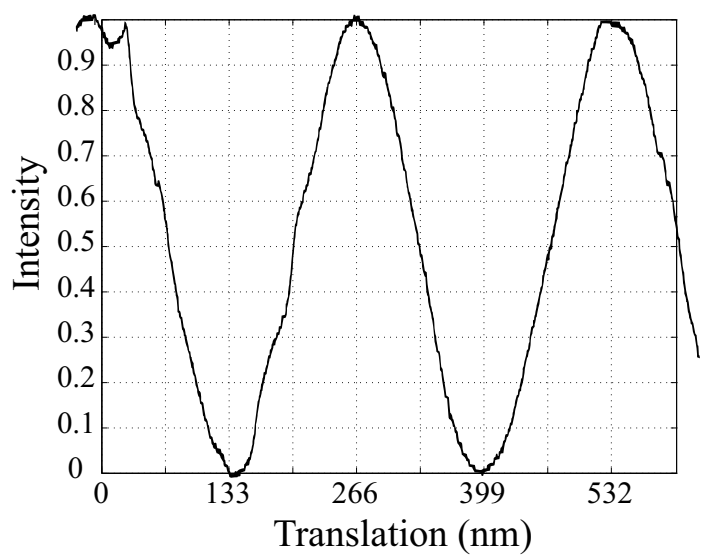

Figure 5: Intensity dependence of the grating translation [8].

magnitude is anticipated to be feasible. This is significantly better than the $0.003^{\circ}$ mentioned in [9].

The LIL-setup used, offers a translational accuracy of only a few nanometers, which, again, is a strong improvement compared with the $17 \mathrm{~nm}$ accuracy, reported in [9]. It is expected that this accuracy can be improved to $1 \mathrm{~nm}$ or better (limited by the translation table), by using a feedback loop from the spot intensity to the translation table controller. The achieved alignment results are sufficiently accurate for the construction of e.g. WPC's.

\section{References}

[1] E. Yablonovitch, "Photonic crystals: semiconductors of light", Scientific American (international edition), vol. 285, pp 47-55, 2001.

[2] J.E.G.J. Wijnhoven and W.L. Vos, "Preparation of photonic crystals made of air spheres in titania", Science, vol. 281, pp. 802-804, 1998.

[3] O. Toader and S. John, "Proposed square spiral microfabrication architecture for large threedimensional photonic band gap crystals", Science, vol. 292, pp. 1133-1135, 2001.

[4] K.M. Ho, C.T. Chan, C.M. Soukoulis, R. Biswas, and M. Sigalas, "Photonic band gaps in three dimensions: new layer-by-layer periodic structures", Solid State Communications, vol. 89, pp. 413416, 1994.

[5] J.G. Fleming and S.Y. Lin, "Three-dimensional photonic crystal with a stop band from 1.35 to 1.95 $\mu \mathrm{m} "$, Optics Letters, vol. 24, pp. 49-51, 1999.

[6] S. Noda, "Three-dimensional photonic crystals operating at optical wavelength region", Physica B, vol. 279, pp. 142-149, 2000.

[7] A. Chutinan and S. Noda, "Effects of structural fluctuations on the photonic bandgap during fabrication of a photonic crystal", Journal of the Optical Society of America B, vol. 16, pp. 240-244, 1999.

[8] Frank J. Soest. A better look at woodpiles. Master's thesis, University of Twente, Faculty of Applied Physics, Integrated Optical MicroSystems group, 2003.

[9] A. Feigel, Z. Kotler, and B. Sfez, "Scalable interference lithography alignment for fabrication of three-dimensional photonic crystals”. Optics Letters, vol. 27, pp. 746-748, 2002. 